\title{
Indicadores da saúde materna e infantil: implicações da décima revisão da Classificação Internacional de Doenças'
}

\author{
Ruy Laurenti e Cássia Maria Buchalla²
}

RESUMO Desde o final da década de 40, quando a Organização Mundial da Saúde assumiu a responsabilidade pelas revisões decenais da Classificação de Causas de Morte, a Classificação passou a incorporar doenças e definições de uso em estatísticas vitais, resultando na Sexta Classificação Internacional de Doenças (CID-6). A mais recente revisão deste documento, a Classificação Estatística Internacional de Doenças e de Problemas Relacionados à Saúde (CID-10), apresenta as mudanças mais significativas desde a CID-6, principalmente no que se refere à área materno-infantil. Entre as mudanças introduzidas pela CID-10 estão a inclusão do tétano obstétrico no capitulo sobre doenças infecciosas, o que facilitará o registro dessa causa de morte materna; a incorporação de novas definições, por exemplo, morte materna tardia; e a redefinição de período perinatal, que a partir da CID-10 começa na $22^{a}$ semana de gestação e termina sete dias completos depois do nascimento. O presente artigo pretende destacar essas mudanças e discutir suas conseqüências para a apresentação e interpretação de indicadores utilizados na avaliação da saúde materno-infantil.

Na segunda metade da década de 40, a Organização Mundial da Saúde (OMS) assumiu a responsabilidade das revisões decenais da Classificação de Causas de Morte, também conhecida como Classificação de Bertillon. Isto ocorreu com a sexta revisão, aprovada em 1948 e posta em uso em 1950. A partir dessa revisão, a Classificação passou a incluir doenças além de causas de morte, e, muito importante, passou também a incorporar as definições

\footnotetext{
1 Uma versão em inglês deste artigo será publicada em um dos próximos números desta revista.

2 Centro Colaborador da Organização Mundial da Saúde para a Classificação de Doenças em Português do Departamento de Epidemiologia da Faculdade de Saúde Pública, Universidade de São Paulo, Av. Dr. Arnaldo, 715, CEP 01246-904, São Paulo, SP, Brasil.
}

de uso em estatísticas vitais. Desde a sexta revisão houve uma expansão da classificação propriamente dita. Atualmente, a rubrica Classificação de Doenças compreende uma família de classificações, assim como as definições e recomendações para uso em estatísticas de saúde, mais particularmente as estatísticas vitais $(1,2)$.

De interesse para a área maternoinfantil, a Classificação Internacional de Doenças (CID) apresenta, entre outras, as definições de nascido vivo, morte fetal, morte infantil, morte materna, morte perinatal, bem como as fórmulas para o cálculo das respectivas taxas e as recomendações para apresentações estatísticas. A nova revisão, CID-10 (3), trouxe as maiores mudanças dentre as revisões sucessi- vas desde a CID-6, com alterações e, principalmente, ampliações em todo o texto, inclusive nos capítulos de interesse das áreas materna e infantil. É importante destacar que algumas definições foram modificadas. $\mathrm{O}$ presente artigo pretende discutir as mudanças introduzidas pela CID-10 e avaliar como elas podem afetar a apresentação e interpretação dos indicadores utilizados em saúde nas áreas materna e infantil. Deve-se notar que na décima revisão o próprio título da CID foi modificado e passou a ser Classificação Estatística Internacional de Doenças e de Problemas Relacionados à Saúde, o que reflete uma maior amplitude de suas finalidades. 


\section{CAPÍTULOS DE INTERESSE PARA A SAÚDE MATERNO-INFANTIL}

No que tange à saúde materna, especificamente aos problemas ligados a gravidez, parto e puerpério, a CID apresenta, desde a sexta revisão, um capítulo denominado "Complicações da gravidez, do parto e do puerpério", renomeado na CID-10 como "Gravidez, parto e puerpério". De interesse para a saúde infantil é o capítulo que engloba as afecções originadas no período perinatal. Porém, as afecções classificadas em outros capítulos podem ser utilizadas, principalmente aquelas referentes às malformações congênitas, nas descrições de freqüência destas doenças em estudos de morbidade ou mortalidade no período perinatal.

\section{Capítulo XV - Gravidez, parto e puerpério (O00-099)}

Este capítulo apresenta atualmente oito agrupamentos, sendo que na CID-9 eram apenas cinco. As categorias passaram de 45 (CID-9) para 75 (CID-10). Ainda que a estrutura deste capítulo não tenha mudado, as alterações introduzidas tornaram mais fácil seu uso para a determinação de índices tanto de morbidade quanto de mortalidade. Vale mencionar que na CID-10 o capítulo XV engloba 25 "categorias vazias", isto é, sem afecções alocadas, o que permite aos usuários fazer expansões visando mais especificações, particularmente em morbidade. Na CID-9 existia apenas uma categoria vazia.

Não é somente a expansão do número de categorias que merece ser citada. Mais importante, o conteúdo do capítulo se tornou mais lógico e satisfatório para os usuários da área da saúde materna, quer em obstetrícia, quer em saúde pública; por exemplo, até a CID-9 era difícil ou impossível classificar uma afecção materna (morbidade ou mortalidade) quando não se sabia exatamente se a complicação era da gravidez, do parto ou do puerpério. A CID-10 trata desta questão, como será discutido mais adiante.
Outra mudança importante é a inclusão da categoria "Tétano obstétrico" no capítulo de doenças infecciosas. Na CID-9, esta categoria não era identificável com o tétano. O tétano obstétrico era incluído no capítulo referente às complicações da gravidez, parto e puerpério, junto com outras afecções infecciosas que podem acompanhar o aborto, a gravidez ectópica ou molar e o puerpério. Esta mudança é pertinente dada a magnitude do problema de mortalidade materna na América Latina $(4,5)$. Assim, a possibilidade de caracterizar o tétano obstétrico nas estatísticas de mortalidade materna será de grande importância para o diagnóstico de situações e elaboração de ações preventivas específicas, o que não era possível na vigência da CID-9. Entretanto, é preciso estar atento para a elaboração das estatísticas de mortalidade materna, pois aos óbitos classificados no capítulo XV deverão ser somados aqueles classificados como "tétano obstétrico" no capítulo I, das doenças infecciosas e parasitárias.

A CID-10 introduziu também três categorias há muito desejadas para uso em análises da mortalidade materna que tornaram possível o reconhecimento de morte materna em situações que antes não podiam ser identificadas. A primeira é "morte obstétrica de causa não especificada", categoria que surge com freqüência em pesquisas descritivas de amostragem (tipo "survey") realizadas com a finalidade de mensurar mortes maternas. A segunda nova categoria se refere à "morte, por qualquer causa obstétrica, que ocorre mais de 42 dias, mas menos de um ano após o parto". Essa categoria será comentada na parte deste artigo que trata de definições. A terceira categoria refere-se à "morte por seqüelas de causas obstétricas diretas". Esta última categoria permite codificar e, portanto, identificar, mortes por causas obstétricas diretas que ocorrem mais de um ano depois do parto. Essa categoria é relevante já que novas aquisições terapêuticas permitem um tempo de sobrevivência maior das vítimas de complicações da gravidez, do parto e do puerpério. Atualmente, a morte pode ocorrer após os 42 dias, e não raro até um ano depois do parto.

\section{Capítulo XVI - Algumas afecções originadas no período perinatal (P00-P99)}

Este capítulo tem origem no capítulo da CID-6 e CID-7 denominado "Doenças peculiares à primeira infância". Na CID-8 o capítulo passou a se chamar "Certas causas de morbidade e mortalidade perinatais"; e, finalmente, na CID-9 e CID-10 recebeu o título mais apropriado de "Algumas afecções originadas no período perinatal". O qualificativo "originadas" significa que as categorias deste capítulo podem ser utilizadas em estudos sobre mortalidade ou morbidade no período perinatal mesmo quando a morte ocorrer depois deste período. De fato, é freqüente seu uso durante os períodos neonatal e infantil, e não raro após um ano de idade.

Da CID-9 para a CID-10 o capítulo XVI sofreu mudanças significativas. A primeira foi a expansão - de 20 categorias na CID-9, o capítulo passou a ter 58 categorias organizadas em 10 agrupamentos na CID-10. Dada a importância que a mortalidade perinatal vem assumindo, principalmente em países desenvolvidos, onde representa a quase totalidade da mortalidade infantil, a expansão deste capítulo facilita a caracterização e detalhamento das causas da mortalidade perinatal.

Alguns agrupamentos merecem destaque neste capítulo. As categorias do agrupamento "Transtornos relacionados com a duração da gestação e com o crescimento fetal" (P05-P08) apresentam definições do tipo glossário. Outro agrupamento importante, particularmente para países em desenvolvimento, é o referente às "Infecções específicas do período perinatal" (P35P39). Apesar de algumas exceções, este agrupamento trata de doenças infecciosas ou parasitárias congênitas ou adquiridas durante o parto. Uma mudança importante nesse agrupa- 
mento foi a exclusão do tétano neonatal, que na CID-10 passou a ser incluído no capítulo sobre doenças infecciosas e parasitárias (categoria A33). Na CID-9 o tétano neonatal era uma subcategoria e, dessa forma, não aparecia rotineiramente nas tabulações como causa de mortalidade neonatal ou infantil. A AIDS causada por transmissão congênita, cuja incidência está aumentando em muitos países, também está incluída no capítulo que trata de doenças infecciosas e parasitárias. A expansão do agrupamento "Transtornos hemorrágicos e hematológicos do feto e do recém-nascido" (P50-P61) - 12 categorias na CID-10 contra apenas duas na CID-9 - possibilita uma especificação mais precisa das patologias descritas nesse agrupamento e é de grande interesse, particularmente para estudos sobre morbidade.

\section{Outros capítulos de interesse}

As causas ligadas à caracterização da morbidade e mortalidade materna, com exceção do tétano obstétrico, estão contidas no capítulo XV. A classificação da morbidade ou da mortalidade por causas originadas no período perinatal, neonatal e infantil é baseada nas categorias que tratam de complicações no período perinatal e no capítulo XVII, "Malformações congênitas, deformidades e anomalias cromossômicas" (Q00-Q99). Isso ocorre principalmente nas áreas onde a mortalidade infantil é baixa e concentrada no período neonatal, particularmente neonatal precoce. Nos países em desenvolvimento, as categorias dos capítulos de causas perinatais e malformações congênitas também são bastante utilizadas na elaboração das estatísticas de morbidade e mortalidade por causas originadas nos períodos neonatal e infantil. Porém, nestes países ainda há um número elevado de causas classificadas no capítulo de doenças infecciosas e parasitárias, bem como no de doenças respiratórias. O capítulo das malformações congênitas na CID-10 está em grande parte baseado nas recomendações da Associação Pediá- trica Britânica e da International Clearinghouse on Birth Defects Monitoring System e apresenta uma grande especificidade conferida por 87 categorias, contra 20 existentes na CID-9.

\section{DEFINIÇÕES EM SAÚDE MATERNO-INFANTIL}

Como mencionado anteriormente, as definições de interesse para estatísticas de saúde, particularmente para estatísticas vitais, estão incorporadas na CID. Algumas dessas definições sofreram alterações na CID-10. Além disso, novas definições foram introduzidas. Este ponto deve ficar claro para os epidemiologistas e administradores de serviços de saúde quando utilizarem indicadores na análise de situações ou na avaliação de programas em saúde materna e infantil. As definições para uso em estatísticas de saúde se encontram no Manual de Instrução (6) da CID-10.

\section{Mortes maternas}

Durante a preparação da CID-10 houve solicitações para que a definição de morte materna (que apareceu pela primeira vez na CID-9) fosse alterada no que se refere ao período de 42 dias após o parto. A proposta era estender o período para até um ano. Esta sugestão não foi incorporada, por razões variadas, entre elas a alegação de que os estudos de tendência seriam afetados. Em contrapartida, uma nova categoria foi incorporada ao capítulo $\mathrm{XV}$, como já anteriormente mencionado: a "Morte materna tardia", por qualquer causa obstétrica, ocorrida mais de 42 dias, mas menos de um ano, depois do parto.

A CID-10 recomenda que as taxas e razões de mortalidade materna continuem sendo calculadas segundo a definição vigente (com limite de 42 dias depois do parto). Aqueles que desejarem calcular uma segunda taxa que inclua as mortes maternas tardias devem ter o cuidado de diferenciar claramente as duas taxas nas apresentações. Além disso, o Manual de Instru- ção da CID-10 recomenda a inclusão em atestados de óbito de questões relacionadas à gravidez em andamento ou à gravidez durante o ano que precedeu a morte (6). Essa recomendação visa a melhorar a qualidade dos dados de mortalidade materna, a fornecer métodos alternativos de coleta de dados sobre morte durante a gravidez, e a encorajar o registro de mortes por causas obstétricas ocorridas mais de 42 dias depois do fim da gravidez. Quanto às causas de mortes maternas, a CID-10 manteve a especificação destas nos dois grupos conhecidos: causas obstétricas diretas e indiretas.

Outro novo conceito incorporado à CID-10 define "Morte relacionada à gravidez" como a morte durante a gravidez ou até 42 dias depois do fim da gravidez, quaisquer que tenham sido as causas da morte. A CID-10 propõe o cálculo de uma razão de mortalidade relacionada à gravidez, na qual o numerador representa as mortes maternas por causas obstétricas diretas e indiretas mais as mortes devidas a quaisquer outras causas (não obstétricas). É claro que esta razão só poderá ser calculada em países que registrarem em atestado de óbito a informação sobre gravidez em andamento ou no ano que precedeu a morte. Tendo em vista que as causas de morte materna são pouco claras nos atestados de óbito, é possível concluir que essa nova definição, bem como o cálculo da razão, objetivam captar os casos de morte materna não caracterizados como tal.

A CID-10 traz ainda definições sobre as razões e taxas referentes à mortalidade materna. Este item recomenda atenção para a inclusão de mortes maternas causadas pelo vírus da imunodeficiência humana (HIV) (B20-B24) ou por tétano obstétrico na razão de mortalidade materna, embora estas causas estejam codificadas em categorias de outros capítulos.

\section{Mortalidade fetal, perinatal, neonatal e infantil}

Com exceção da definição de período perinatal, que será comentada 
abaixo, as definições de "nascimento vivo"; "óbito fetal"; "peso ao nascer"; "baixo peso ao nascer"; "peso muito baixo ao nascer"; "peso extremamente baixo ao nascer"; "idade gestacional"; "pré-termo"; "pós-termo"; e "período neonatal" não foram modificadas na CID-10. As definições de taxas e razões de mortalidade fetal e taxas de mortalidade neonatal e infantil também não sofreram modificações.

\section{Período perinatal e mortalidade perinatal}

A definição de período perinatal foi modificada na CID-10. Conseqüentemente, a interpretação do termo "morte perinatal" e das taxas respectivas deverá sofrer mudanças. Considerando que a mortalidade perinatal é um indicador cada vez mais utilizado para medir saúde infantil e recomendado também para a avaliação de saúde materna, comentários mais extensos sobre o porquê destas mudanças serão feitos abaixo.

Peller (7) propôs o termo perinatal em 1940, pois considerava necessário analisar como uma "unidade estatística" a soma dos nascidos mortos (perdas fetais tardias) com a morte durante os sete primeiros dias de vida. Para os estatísticos, o uso desse conceito facilitou as comparações de dados em âmbito internacional. Clínicos e epidemiologistas perceberam que os dois componentes da mortalidade perinatal estão sujeitos aos mesmos fatores causais, de natureza pré-natal e intranatal. Embora claro, o conceito de período perinatal e das mortes ocorridas nesse período, como proposto por Peller, nem sempre era corretamente aplicado, particularmente em relação ao que se denominava de natimorto ou nascido morto. $\mathrm{O}$ conceito de nascido morto variava de país para país, e as legislações vigentes nem sempre definiam o termo ou exigiam seu registro obrigatório. Depois de assumir a responsabilidade pelas sucessivas revisões da Classificação Internacional de Doenças, a OMS incorporou na CID-7 (1955) as definições de nascido vivo e de morte fetal, facilitando um consenso internacional sobre "nascido morto" como sinônimo de "morte fetal tardia", ou morte ocorrida com 28 semanas ou mais de gestação (8). A CID-7 previu ainda o registro e tabulação de mortes fetais como tendo acontecido nos períodos "precoce", "intermediário" ou "tardio", de acordo com os mesmos períodos de gestação aplicados aos nascidos vivos. $\mathrm{O}$ termo aborto não foi definido. A recomendação da CID-7 foi para que o termo "nascido morto" fosse usado como sinônimo de "morte fetal tardia", e dessa maneira pudesse ser utilizado nos países que elaboravam estatísticas sobre nascidos mortos.

A CID-8 incorporou a definição de período perinatal como o período que se estende do início da $28^{\mathrm{a}}$ semana de gestação até o sétimo dia de vida (9). Assim, o termo perinatal e seu respectivo conceito, proposto por Peller (7), passou a ser utilizado internacionalmente. A CID-9 procurou outros elementos para caracterizar o início do período perinatal, passando a incluir peso (1000 g ou mais) como o principal fator de definição, seguido de idade gestacional correspondente (28 semanas) quando o peso ao nascer não estivesse disponível, ou comprimento do corpo $(35 \mathrm{~cm}$, medidos do ponto mais alto da cabeça até os calcanhares) (10). Como na década de 70 neonatologistas e obstetras já consideravam que o período perinatal deveria iniciar antes da $28^{\text {a }}$ semana de gravidez, a CID-9 estimulava que as estatísticas perinatais nacionais incluíssem todos os fetos e recém-nascidos com peso mínimo de $500 \mathrm{~g}$, que corresponde à idade gestacional de 22 semanas. Para as comparações internacionais, o limite continuou sendo $1000 \mathrm{~g}$ ou mínimo de 28 semanas de gestação.

Na CID-10, o período perinatal passa a iniciar em 22 semanas completas (154 dias) de gestação, quando o peso de nascimento é normalmente $500 \mathrm{~g}$, terminando sete dias completos após o nascimento. Essa mudança trará conseqüências para as estatísticas de natimortalidade e mortalidade perinatal. As perdas fetais a partir da $22^{\mathrm{a}}$ semana serão acrescidas ao numerador para o cálculo de taxa de mortalidade perinatal. Essa taxa, portanto, deverá aumentar, com conseqüências para estudos de tendências. Algumas publicações científicas já vêm fazendo considerações sobre essa situação (11-14). O termo nascido morto ou natimorto passará a ser identificado provavelmente com as perdas fetais a partir da $22^{\mathrm{a}}$ semana de gestação, ou quando o feto atinge peso de $500 \mathrm{~g}$. Essas perdas fetais, provavelmente, também serão denominadas "perdas fetais tardias". Assim, uma nova maneira de calcular a taxa de natimortalidade estará disponível e será um indicador útil para avaliar a atenção à saúde materna.

Finalmente, é importante ressaltar um aspecto relacionado à comparabilidade internacional das taxas de mortalidade perinatal e natimortalidade. As legislações dos países variam quanto à obrigatoriedade da inscrição das perdas fetais no registro civil: em alguns países há disposições legais para registrar todas as perdas fetais; em outros, as perdas a partir da $20^{\mathrm{a}}$ semana são registradas. Um terceiro caso é a obrigatoriedade de registro apenas para os natimortos. Nesses casos, é comum que os natimortos sejam considerados como perdas fetais tardias, isto é, aquelas a partir do início da $28^{a}$ semana de gestação. Como é difícil alterar a legislação, é possível prever dificuldades no cálculo de natimortalidade e mortalidade perinatal a partir da nova definição de período perinatal incorporada à CID-10. Esta questão deverá ser levada em conta por epidemiologistas e administradores de saúde na área materno-infantil. $\mathrm{Na}$ Região das Américas, onde a Organização Panamericana da Saúde mantém um projeto para a redução da mortalidade materna (5), é preciso discutir este problema e propôr soluções, inclusive mudanças na legislação, já que esses indicadores são extremamente úteis para a avaliação de programas na área da saúde maternoinfantil. 


\section{REFERÊNCIAS}

1. Laurenti R. Análise da informação em saúde: 1893-1993. Cem anos da classificação internacional de doenças. Rev Saude Publica 1991;25(6):407-417.

2. Laurenti R. Décima revisão da classificação internacional de doenças e de problemas relacionados à saúde (CID-10): a revisão do final do século. Bol Oficina Sanit Panam 1995; 118(3):273-280.

3. Organização Mundial da Saúde. Classificação internacional de doenças e problemas relacionados à saúde. Décima revisão. São Paulo: Centro Colaborador da OMS para a Classificação de Doenças em Português; 1993.

4. Laurenti R. Maternal mortality in Latin American urban areas: the case of S. Paulo, Brazil. Bull Pan Am Health Organ 1993;27(3):205-214.

5. Organización Panamericana de la Salud. Plan de acción regional para la reducción de la mortalidad materna en las Americas. Washington, DC: XXIII Conferencia Sanitaria Panamerica; 1990.
6. Organização Mundial da Saúde. Classificação estatística internacional de doenças e de problemas relacionados à saúde. Décima revisão. Manual de instrução (volume 2). São Paulo: Centro Colaborador da OMS para a Classificação de Doenças em Português; 1994.

7. Peller S. Proper delineation of the neonatal period in perinatal mortality. Am J Public Health 1965;55:1005-1011.

8. Organização Panamericana da Saúde. Manual da classificação internacional de doenças, lesões e causas de óbito. Sétima revisão. Washington, DC: OPS; 1964. [Versão em português.]

9. Organização Panamericana da Saúde. Manual da classificação internacional de doenças, lesões $e$ causas de óbito. Oitava revisão. Washington, DC: OPS; 1969. [Versão em português.]

10. Organização Mundial da Saúde. Manual da classificação internacional de doenças, lesões e morte. Nona revisão, 1975. São Paulo: Centro Colaborador da OMS para a Classificação de Doenças em Português; 1978.
11. Wigglesworth JS. Classification of perinatal deaths. Soz Praventivmed 1994;39:11-14.

12. Gissler M, Ollila E, Teperi J, Hemminki E. Impact of induced abortions and statistical definitions on perinatal figures. Paediatr Perinat Epidemiol 1994;8:391-400.

13. Gourbin C, Masuy-Stroobant G. Are live and stillbirths comparable all over Europe? Legal definitions and vital registration data processing. International Institute for Vital Registration and Statistics, Technical paper no. 58, August, 1994: 58.

14. Cartlidge PH, Steward JH. Effect of changing the stillbirth definition on evaluation of perinatal mortality rates. Lancet 1995;346:486-488.

Manuscrito recebido em 30 de novembro de 1995. Aceito em 4 de junho de 1996 para publição em português e em inglês.

ABSTRACT Since the end of the 1940s, when the World Health Organization assumed responsibility for the decennial revisions of the Classification of Causes of Death, the Classification came to include diseases and definitions of use in vital statistics, resulting in the Sixth International Classification of Diseases (ICD-6). The most recent revision of this work, the International Statistical Classification of Diseases and Related Health Problems (ICD-10), is more significantly different than any revision since ICD-6, especially in the area of maternal and child health. Among the changes introduced in ICD-10 are the inclusion of obstetrical tetanus in the chapter on infectious diseases, which will facilitate the recording of this cause of maternal death; the incorporation of new definitions, such as late maternal death; and the redefinition of the perinatal period, which ICD-10 defines as starting at 22 completed weeks of gestation and ending 7 completed days after birth. This article seeks to highlight these changes and to discuss their consequences for the presentation and interpretation of indicators used in the evaluation of maternal and child health. 